\title{
THE HELICIAL FLUX ROPE STRUCTURE OF SOLAR FILAMENTS
}

\author{
D. M. Rust \\ JHU/Applied Physics Laboratory, 11100 Johns Hopkins Road, Laurel, MD 20723-6099, USA
}

\begin{abstract}
According to J. B. Taylor's relaxation theory for magnetized plasmas, helical flux ropes should be the fundamental building blocks of magnetism in the solar atmosphere. Although flux ropes are clearly ejected from the Sun, there has been some controversy about whether they exist in the sun's atmosphere before ejection. The issue is important because of the need to understand how eruptions are initiated. A clearer picture of solar flux ropes is emerging with recent high-resolution observations of solar filaments. Their twist and writhe are frequently detectable. This report describes some examples of filaments that suddenly writhe (kink) and do not escape the sun. The observations of November 1, 2001 and May 27, 2002 both appear to show the sudden eruption of a filament with probable transformation of some internal twist into a writhe of approx. +1 . Also described is one event in which a well observed filament did erupt. It is associated with a magnetic flux rope detected with the NEAR spacecraft magnetometer.
\end{abstract}

\section{INTRODUCTION}

Berger and Field (1984) proposed that magnetic helicity, $H_{m}=\int \mathbf{B} \cdot \mathbf{A} \mathrm{dV}$, might find useful applications in astrophysics. Their proposal built on Taylor's $(1974 ; 1986)$ conjecture that $H_{m}$ is conserved in closed volumes, even in the non-ideal MHD approximation. The validity of Taylor's conjecture is convincing (Berger 1999) in circumstances that can include solar active regions with magnetic field reconnections taking place. At first, little attention was paid to these developments. However, House and Berger (1987) discussed the eruption of a helical prominence using $H_{m}$ conservation to describe the transfer of twist from one part of the prominence to another. Seehafer (1990) noticed that active regions tend to have left-handed helical currents in the northern hemisphere and positive ones in the south, regardless of phase in the 22-yr solar magnetic cycle. He also pointed out that $h_{m}$, the magnetic helicity density, is proportional to current helicity, $h_{c}$, which can be inferred from vector magnetograms, so $h_{m}$ must show a hemispherical preference, as well. Next, Martin et al. (1992) reported that high-latitude H $\square$ filaments (Figure 1) also show a hemispherical preference, according to their morphology, with one type predominating in the north and the mirror image type predominating in the south. The hemispherical preference holds regardless of the phase of the solar cycle. It is also the same for both even- and odd-numbered cycles.

Rust and Kumar (1993) proposed that magnetic helicity flows from the photosphere to the corona and filaments by a process of 'helicity charging,' and Rust and Kumar (1993) developed a flux rope model for filaments in which a filament's morphology depends on whether it is formed by magnetic flux with right-handed or lefthanded magnetic helicity. Rust (1994) and Bothmer and Schwenn (1994) showed that the chirality (sign of $H_{m}$ ) of interplanetary magnetic clouds (Burlaga et al., 1982) associated with filament eruptions from the north (south) is usually negative (positive). The fields of magnetic clouds can be fitted quite satisfactorily with the Lundquist (1950) solution for a cylindrical force-free flux rope (Lepping et al., 1990). Because magnetic helicity in a flux rope should be conserved as it travels from the Sun to Earth, it is plausible, then, that eruptive filaments and the corona surrounding them also have a flux rope structure. 


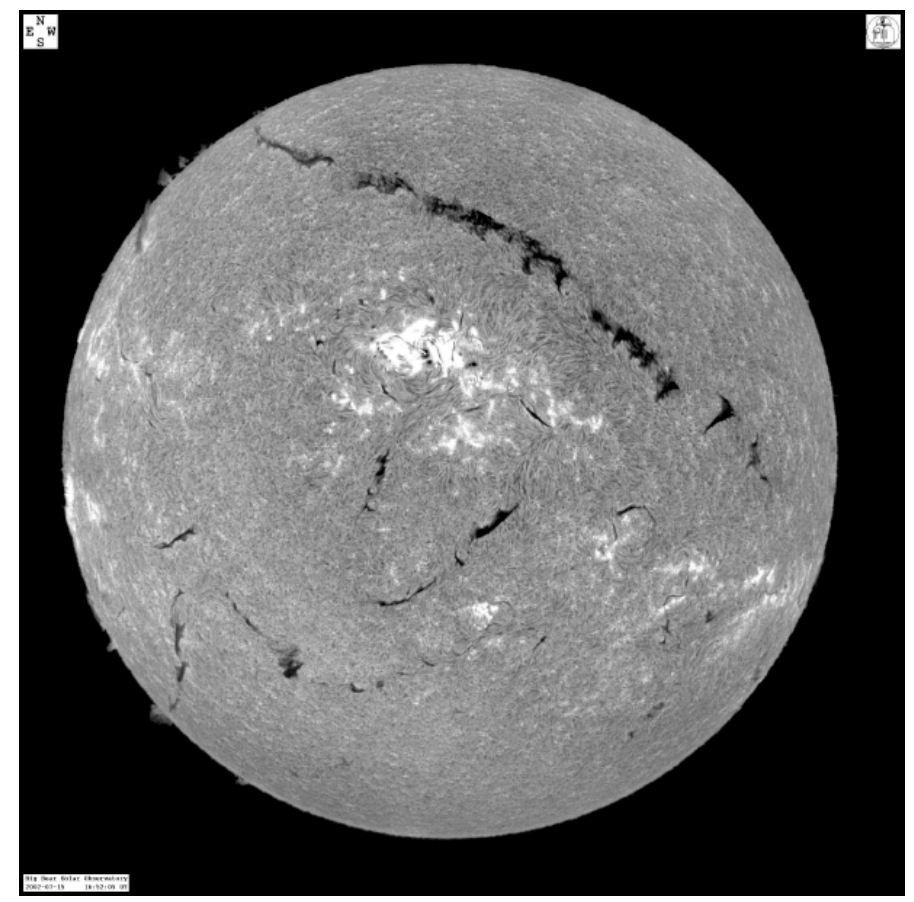

Fig. 1. $\mathrm{H} \square$ picture of the sun on July 15,2002 . Note the extraordinarily long filament (BBSO image)

Low (1994) suggested that helicity in the corona could be best explained by emergence of already twisted flux from beneath the photosphere. Finally, Rust (1994) and Low (1996) used magnetic helicity conservation in conceptual models linking helicity spawned by the dynamo with transfer of helicity to the corona, where it would accumulate and be eventually shed into interplanetary space by coronal mass ejections (CMEs). Now, an expanding number of works aim to quantify $H_{m}$ in solar active regions, CMEs, and magnetic clouds. Much of the recent work is well represented in the book edited by Brown, Canfield and Pevtsov (1999). See especially Berger's (1999) paper for details of the theoretical application of helicity conservation in solar physics.

\section{MAGNETIC HELICITY IN FILAMENTS}

$H_{m}$ in filaments can be obtained from solar observations only by indirect means, e.g., by estimating the magnetic flux threading the filament and measuring the number of turns. In filaments, $H_{m}$ can be estimated from the formula: $H_{m}=(T w+W r) \square^{2}$, where the twist $T w$ is the number of turns of the field about the axis; the writhe $W r$ is the number of times the axis loops on itself, i.e., it is the helicity of the axis itself (Berger, 1999); and $\square$ is the magnetic flux in the filament.

Prominences, which are just filaments on the limb of the sun, are highly visible components of many CMEs, and their helical structure can be seen often in images from the LASCO coronagraph on the SOHO spacecraft. We can compare the helicity in eruptive prominences with that in the corresponding magnetic clouds. Just what part of a magnetic cloud corresponds to the eruptive prominence is not clear, but right-handed clouds are associated statistically with the eruption of right-handed filaments, similarly for left-handed clouds and filaments (Bothmer and Rust, 1997). Prominences have magnetic fields of about $10 \mathrm{G}$ (Rust, 1967) and a twist plus writhe of $\sim 2$ upon eruption (see the example below). The cross sectional area is about $5 \times 10^{18} \mathrm{~cm}^{2}$, so the magnetic helicity, $H_{m}$ is about $0.5 \times 10^{40} \mathrm{Mx}^{2}$. This is considerably less than the average helicity for a magnetic cloud: $2 \times 10^{42} \mathrm{Mx}^{2}$ (DeVore, 2000). Most of the helicity in clouds must originate in the corona, probably in the dark cavity surrounding the prominence. Assuming an average twist there of 2, we need to find $10^{21} \mathrm{Mx}$ of flux to associate with the part of the corona that erupts. With an estimate of $3 \mathrm{G}$ for the coronal field, we get a cross-sectional area with a radius of about $100,000 \mathrm{~km}$, which is consistent with observations of coronal cavities (Serio et al., 1978). On the basis of the photospheric flux under eruption-associated transient coronal holes, Lepping et al. (1997) calculated that the coronal magnetic flux associated with clouds is about $4 \times 10^{20} \mathrm{Mx}$. There is a great deal of uncertainty in these estimates and measurements of coronal magnetic fields will be needed to resolve it.

A controversy has raged in solar physics about whether filaments are actually flux ropes. It has long been conceded that many prominences have a helical structure when they are erupting. Everyone is familiar with the 


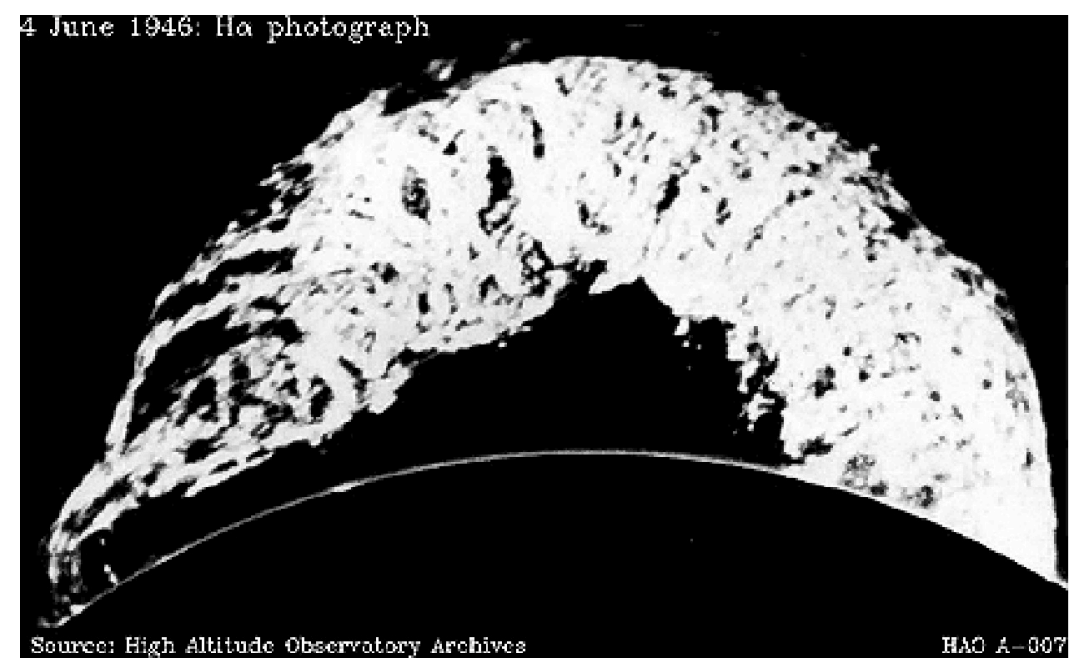

Fig. 2. Photograph of the giant eruptive prominence of June 4, 1946. Note the coiled appearance.

eruptive prominence photographed at the High Altitude Observatory Climax Station on June 4, 1946 (Figure 2). But some have argued that the helical structure is created only during the eruption process (Antiochos and DeVore, 1999; Gosling, 1999). With the deepening understanding of magnetic helicity on the sun and with improved observations, I think it is now possible to answer the following key questions: Before eruption, is there definitive evidence that filaments are flux ropes, as one would expect from Taylor relaxation, and what is the relationship between the helical properties of the filament flux rope and those of the corresponding magnetic cloud?

\section{OBSERVATIONS OF TWISTED AND WRITHING FILAMENTS}

An $\mathrm{H} \square$ movie of an active region with a filament showing helical signatures was obtained on August 17, 1999 at the Big Bear Solar Observatory. Figure 3 shows a typical frame of the movie, which captured key features of the filament with unusually good seeing conditions. Although the filament showed twisting motions, it did not erupt. It had one end rooted in a sunspot whose chromospheric fibril structure makes it look like the spot was rotating counterclockwise. This is a well-known signature of an end-on view of a flux rope with negative (left-handed) helicity (Nakagawa et al., 1971; Rust and Martin, 1994).

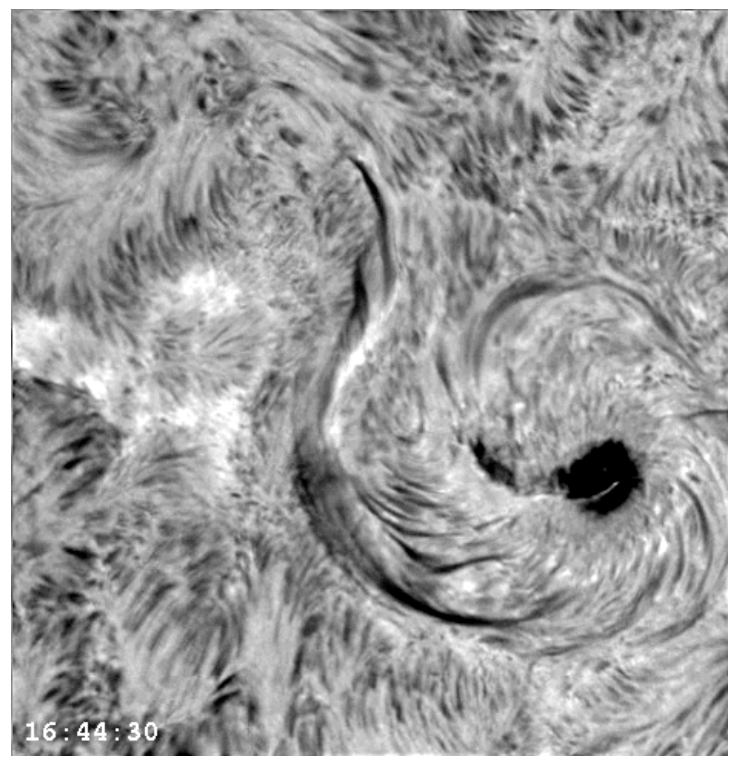

Fig. 3. Active Region NOAA 8668 on August 17, 1999, including a filament with left-handed twist (BBSO image). 


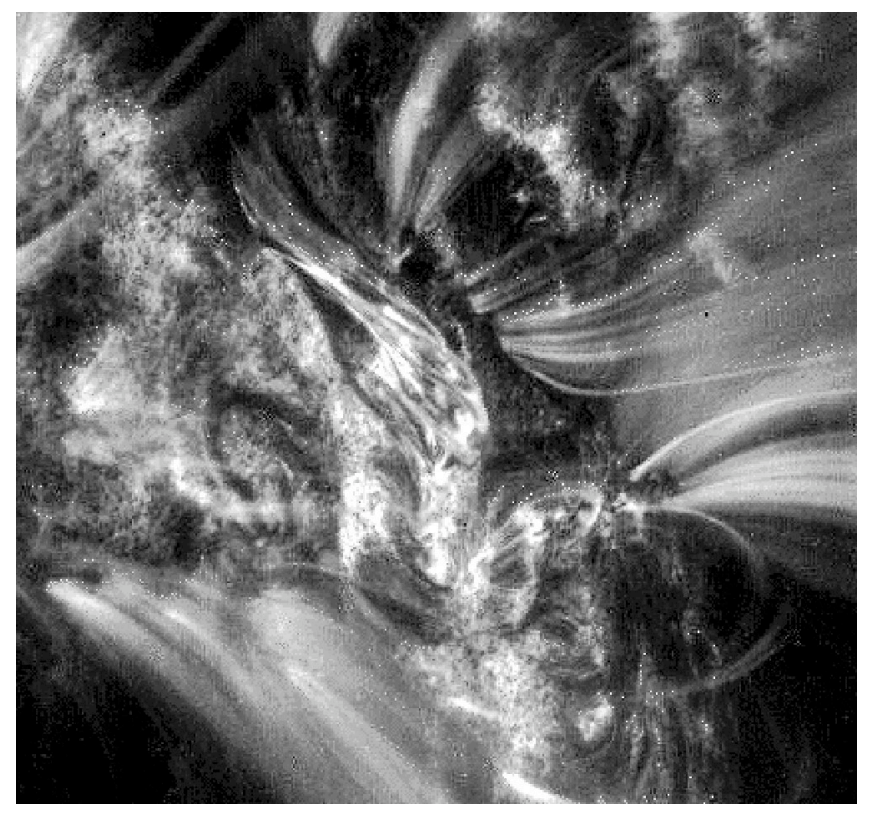

Fig. 4. Activated, but stable filament seen in $171 \AA$ on September 7, 2001 (TRACE image).

The filament's sinuous morphology indicates that it also had a left-handed field: it looks like a reverse S. As Rust and Kumar (1994) showed, a twisted flux rope will writhe and take on the reverse $\mathbf{S}$ shape if the amount of negative twist in it exceeds the limit for the kink instability, which occurs when $2 \square T w R / L>0.581$. Tw is the number of turns from end to end, and $2 R / L$ is the 'aspect ratio,' the width of the $\mathbf{S}$ divided by it length. From Figure 3 we can estimate an aspect ratio of $1 / 4$ and a twist $T w$ of 1 , so that $2 \square T w R / L=0.78$. This result is consistent with the observed distribution of X-ray sigmoids, which peaked at 0.65 .

That the twist $T w$ is left-handed and about equal to unity is evident from the movie, which shows material flowing around the axis as though following the grooves of a left-handed screw. Taken together, sunspot whorl, filament $\mathbf{S}$ shape, and filament fine structure are strong evidence that the field of the filament was a left-handed flux rope rooted in the sunspot. According to Chae et al. (2001), motions in the photosphere could account for the transfer of negative helicity into the filament and corona from the active region. $\mathrm{H} \square$ observations have shown the helical structure of filaments for nearly a century. The new generation of high-resolution observations, however, provides the clearest and most convincing evidence of flux rope structure in filaments. The TRACE mission provides on their web page, vestige.lmsal.com/TRACE/POD/TRACEpod.html, a library of movies, many of which show filaments in various stages of activation and eruption. The particular advantage of the TRACE movies is that they are able to show the filament material before and during eruption much more clearly than $\mathrm{H} \square$ does. The reason is that rapidly moving material is not visible through the narrow-band $\mathrm{H} \square$ filters whereas the TRACE filters are so wide that the Doppler effects cannot shift the wavelength of the emitting material out of the passband.

One sequence (Figure 4), obtained in the $171 \AA$ line of Fe IX on September 7, 2001, demonstrates the twisted flux rope structure particularly well. It shows a filament with bright and dark threads wrapped around each other like a cable with silver and copper strands. Judging from the bright thread curving over the top of the filament, I estimate that the strands make one half of a full turn from one end of the filament to the other. Although the strands appear to be straining to break loose, the filament did not erupt, nor would one expect it to, because instability should not occur for $T w<0.75$, since the filament's aspect ratio looks to be about $1 / 4$.

Another example of twisted flux rope structure is seen in Figure 5. The movie of this event in active region NOAA 9682 on November 1, 2001 shows that the filament started to erupt but failed to leave the field of view of the TRACE telescope. Many filaments undergo "frustrated" eruptions like this one. The bright threads of the filament (see the upper right quadrant of Figure 5) curl around for about one turn in the right-handed sense. The frustrated eruption may in reality be just the development of a kink (writhe) in a filament that became twisted beyond the kink limit. When a twisted flux rope develops a kink instability, it simply transfers some of its helicity from twist to writhe. We do not know how rapidly this happens, but it is probably fairly fast. The frustrated eruptions I studied showed transformations of filaments from linear structure to $W r \approx 1$ structure in 15 to $30 \mathrm{~min}$. Despite the rapidity of the event, there was no associated coronal mass ejection. 


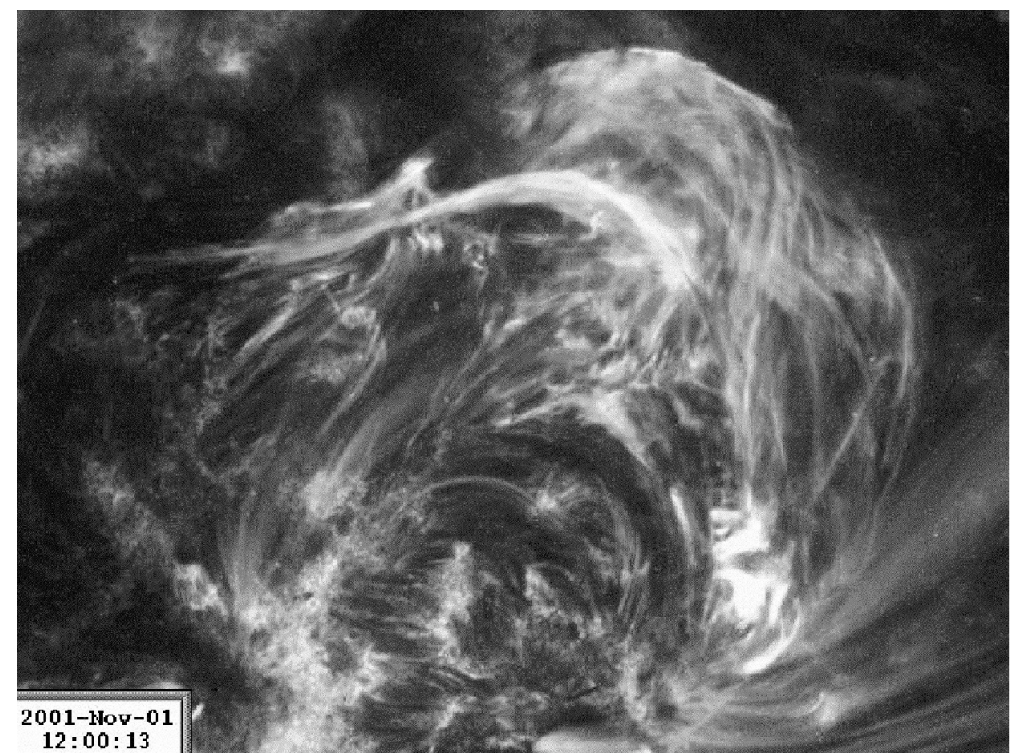

Fig. 5. Fe IX image of a filament undergoing a frustrated eruption on November 1, 2001 (TRACE image).

Figure 6 shows another frustrated filament eruption which, like many such events, also involved a flare. The figure shows a filament just before eruption and then in various stages of transformation as it writhed to become a loop with writhe equal to +1 . The rightmost image shows the filament at its highest point, where its rapid initial growth stopped abruptly. At that point the filament threads began to brighten and break away from the main body. The twist in the individual threads is difficult to see but it appears to be right-handed. There was no associated CME.

\section{A POSSIBLE FILAMENT AT 1 AU}

In a search for the interplanetary counterparts of particularly well-observed filament eruptions and CMEs, M. Andrews, B. Anderson, T. Mulligan and I (manuscript in preparation) studied magnetic clouds recorded by the Near Earth Asteroid Rendezvous (NEAR) spacecraft. NEAR provided magnetometer observations with an accuracy of $1 \mathrm{nT}$, which is sufficient to characterize magnetic clouds. NEAR varied in heliocentric distance between 1 and $1.8 \mathrm{AU}$, at angular separations from the Earth-Sun line of $0^{\circ}$ to $180^{\circ}$. It was often more-or-less in quadrature with Earth, i.e., its position has often been ideal for sampling the CMEs seen with the SOHO telescopes. We searched for possible CME counterparts and signatures of prominence eruption for the NEAR magnetic clouds. We found a general agreement in the inferred magnetic chirality between the solar and NEAR events. Here I give one example event.

The disturbance in the IP magnetic field at NEAR started late on August 19, 1997 and continued for about $30 \mathrm{~h}$ (Figure 7). At 0600 on August 20, in the midst of the disturbance, there was an unusually sharp change in the field. As shown on the bottom of Figure 7, it had the characteristic signature of a flux rope: a relatively strong field with low variance and a characteristic rotation in one axis. The short duration was unusual, only about $3.5 \mathrm{~h}$, and except in the flux rope, this event showed no overall rotation in the field.

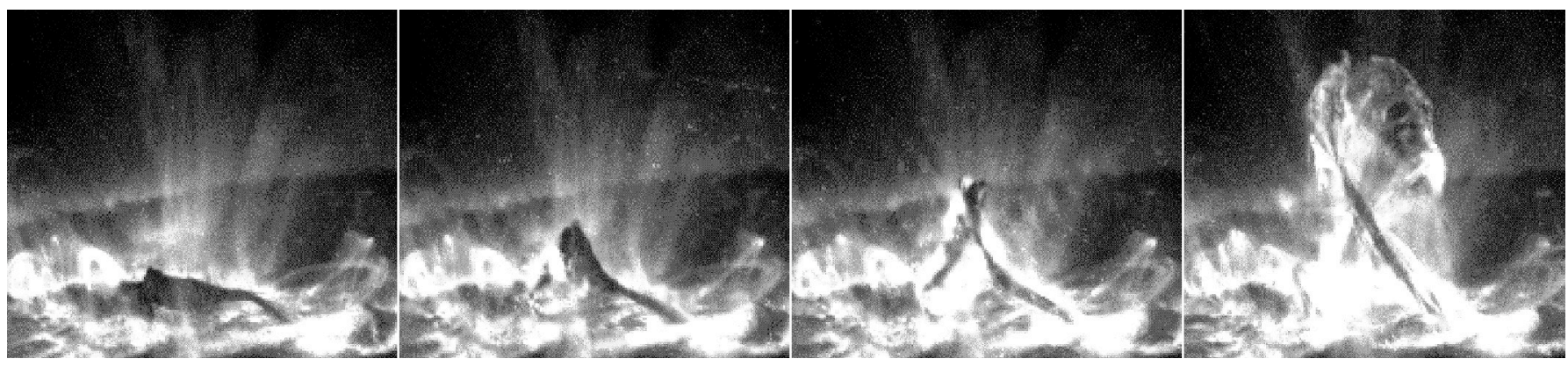

Fig. 6. Writhing of a dark filament on May 27, 2002, at 1800 UT (TRACE images) 


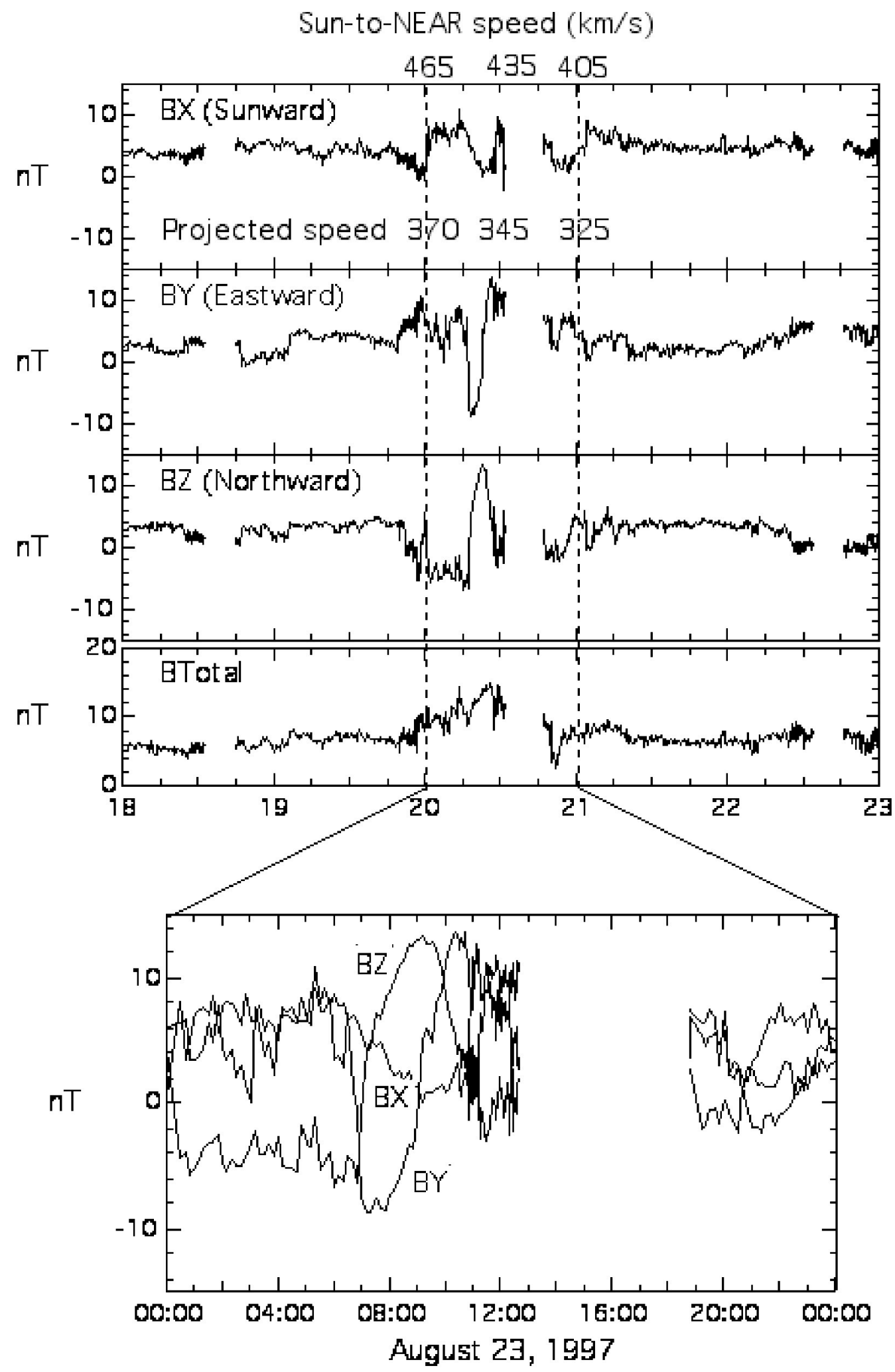

Figure 7. NEAR magnetometer data in NEAR-Ecliptic-Polar coordinates for the period August 18 to 23 (days 230 to 235) 1997. At this time NEAR was $57^{\circ}$ west of the Earth-Sun line at a heliocentric distance of $1.78 \mathrm{AU}$. The timeof-flight speeds for various features are indicated at the top of the figure. The spiked feature at $435 \mathrm{~km} / \mathrm{s}$ is expanded in the bottom panel. Its field rotates from west to east and has a northward axial field. Mulligan's model fit indicates that this is a right-handed flux rope. 

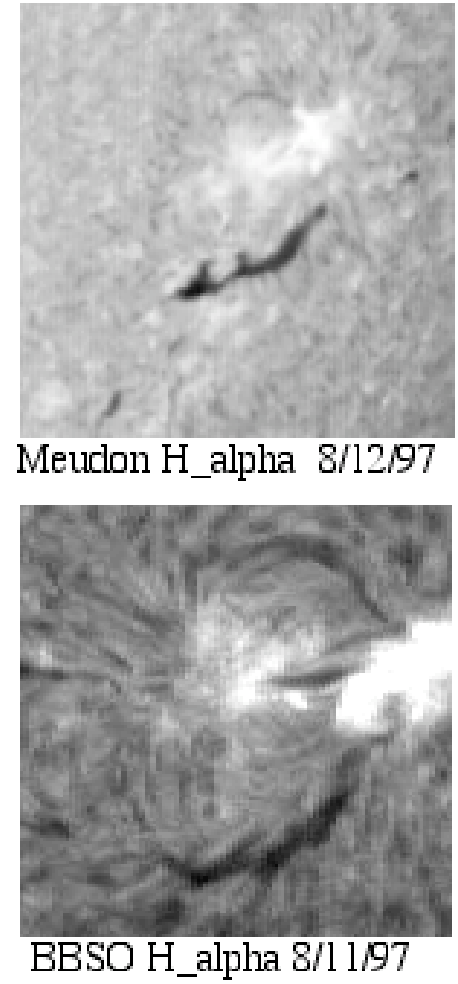
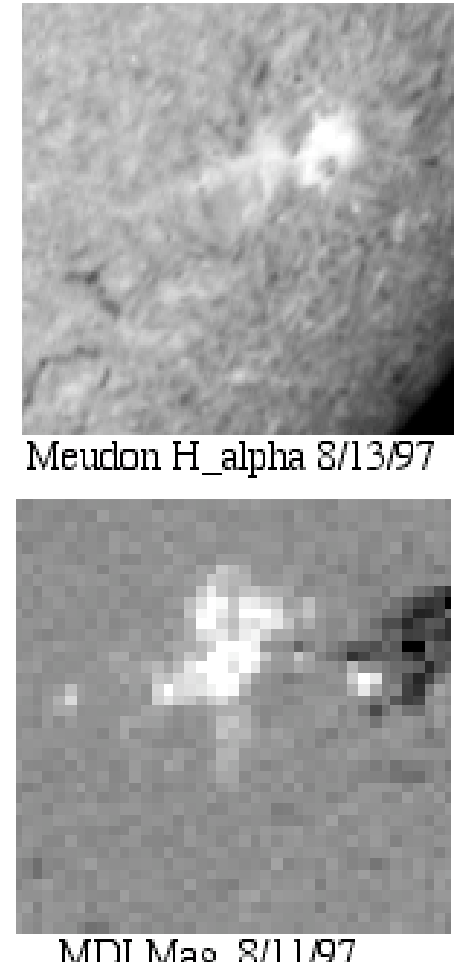

MDI Mag. 8/11/97

Fig. 8. H $\mathrm{H}$ images on August 13, 1997 showing the filament that erupted to produce the magnetic cloud detected by the NEAR magnetometer on August 20. From the Big Bear Solar Observatory image and the MDI magnetogram we deduced that the flux rope associated with the filament had a right-handed twist.

There was only one likely CME candidate in the acceptance window of $9-15$ August 1997 . A streamer blowout without any obvious flux rope structures appeared in the LASCO C2 field of view at 0800 UT on August 13. On the same day, the SOHO/EIT $195 \AA$ images showed the coronal dimming and sigmoid brightening, at about $\mathrm{S} 20^{\circ} \mathrm{W} 48^{\circ}$, that is the characteristic signature of a CME launch. GOES recorded a B2.1 X-ray event at $0642 \mathrm{UT}$. Figure 8 shows that a large filament disappeared there between 0730 UT on August 12 and 0730 UT on August 13. From the H $\square$ image and the magnetogram on August 11 we inferred that it had a right-handed helical field, according to the interpretation method of Rust and Kumar (1994). The NEAR flux rope was also right-handed, according to a model fit to it by T. Mulligan (pvt. comm, see also (Mulligan et al., 1999)). Our tentative conclusion is that NEAR did intercept a flux rope corresponding to the filament only.

\section{CONCLUSIONS}

From Taylor's theory of relaxation in non-ideal MHD, we expect magnetic helicity to be conserved and flux ropes to form on the sun. Few would dispute the fact that magnetic clouds, which are ejected from the sun in CMEs, are flux ropes. Nor would many dispute the fact that eruptive prominences, such as the one shown in Figure 2, are flux ropes. However, some authors, e.g., (Antiochos and DeVore, 1999; Gosling, 1999), have disputed the general assumption that the ejected flux ropes were present in the corona before eruption. The issue is an important one because we seek to understand the physical mechanisms of coronal mass ejections. The observations discussed in this paper show filaments that look like flux ropes, twist like flux ropes, writhe like flux ropes, erupt like flux ropes. We conclude that they are flux ropes. From the examples shown here, we can also conclude that filaments with right/left-handed twist show right/left-handed writhe. That is, twist appeared to be transformed into writhe.

The observations of November 1, 2001 and May 27, 2002 both appear to show the sudden eruption of a filament with probable transformation of some internal twist into a writhe of approx. +1 . Careful examination of the LASCO coronal movies established that no CME's occurred in either case. This has important and interesting consequences, namely, that the helicity in the region did not escape. According to Low's (1996) model it would available to help power CME's after further helicity charging. 
Filaments that erupt from the northern/southern hemisphere have been associated statistically with magnetic clouds with negative/positive helical flux ropes. New studies, such as the one with the NEAR and SOHO data described here and the one by Webb et al. (2000), are beginning to associate particular, well-characterized interplanetary magnetic cloud flux rope signatures with the signatures of flux ropes at the sun. The emerging conclusion is that not only are filaments flux ropes, they certainly have the same chirality as their corresponding magnetic clouds. The next task is to better characterize the flux rope twist and writhe so that the conditions for eruptions can be discovered. Systematic measurements of Tw and Wr are needed. The plethora of high-resolution movies now becoming available will make this possible.

\section{ACKNOWLEDGEMENTS}

NASA funded this work under grants NAG5-7921 and NAG 5-11584. I thank the NEAR, Big Bear Solar Observatory and the TRACE teams for making their data so freely available. The Transition Region and Coronal Explorer, TRACE, is a mission of the Stanford-Lockheed Institute for Space Research (a joint program of the Lockheed-Martin Advanced Technology Center's Solar and Astrophysics Laboratory and Stanford's Solar Observatories Group), and part of the NASA Small Explorer program.

\section{REFERENCES}

Antiochos, S., and C.R. DeVore, The role of helicity in magnetic reconnection: 3D numerical simulations, in Magnetic Helicity in Space and Laboratory Plasmas, edited by M.R. Brown, R.C. Canfield, and A.A. Pevtsov, pp. 157-166, Amer. Geophys. Union, Washington, DC, 1999.

Berger, M., Magnetic helicity in space physics, in Magnetic Helicity in Space and Laboratory Plasmas, edited by M.R. Brown, R.C. Canfield, and A.A. Pevtsov, pp. 1-9, Amer. Geophys. Union, Washington, DC, 1999.

Berger, M.A., and G.B. Field, The topological properties of magnetic helicity, J. Geophys. Res., 147, 133-148, 1984

Bothmer, V., and D.M. Rust, The field configuration of magnetic clouds and the solar cycle, in Coronal Mass Ejections, edited by N. Crooker, J.A. Joselyn, and J. Feynman, pp. 137-146, Amer. Geophys. Union, Washington DC, 1997.

Bothmer, V., and R. Schwenn, Eruptive prominences as sources of magnetic clouds in the solar wind, Space Sci. Rev., 70, 215-225, 1994.

Brown, M.R., R.C. Canfield, and A.A. Pevtsov, Magnetic Helicity in Space and Laboratory Plasmas, Amer. Geophys. Union, Washington DC, 1999.

Burlaga, L.F., L. Klein, N.R.J. Sheeley, et al., A magnetic cloud and a coronal mass ejection, Geophys. Res. Lett., 9, 1317-1320, 1982.

Chae, J., H. Wang, J. Qiu, et al., The formation of a prominence in active region NOAA 8668. I. SOHO/MDI observations of magnetic field evolution, Astrophys. J., 560, 476-489, 2001.

DeVore, C.R., Magnetic helicity generation by solar differential roation, Astrophys. J., 539, 944-953, 2000.

Gosling, J.T., The role of reconnection in the formation of flux ropes in the solar wind, in Magnetic Helicity in Space and Laboratory Plasmas, edited by M.R. Brown, R.C. Canfield, and A.A. Pevtsov, pp. 205-212, Amer. Geophys. Union, Washington, DC, 1999.

House, L.L., and M.A. Berger, The ejection of helical field structures through the outer corona, Astrophys. J., 323, 406-413, 1987.

Lepping, R.P., J.A. Jones, and L.F. Burlaga, Magnetic field structure of interplanetary magnetic clouds at 1 AU, $J$. Geophys. Res., 95, 11957-11965, 1990.

Lepping, R.P., A. Szabo, C.E. DeForest, et al., Magnetic flux in modeled magnetic clouds at 1 AU and some specific comparisons to associated photospheric flux, in Correlated Phenomena at the Sun, in the Heliosphere, and in Geospace, pp. 163-170, ESA SP-415, Paris, 1997.

Low, B.C., Magnetohydrodynamic processes in the solar corona: flares, coronal mass ejections and magnetic helicity, Phys. Plasmas, 1 (5), 1684-1690, 1994.

Low, B.C., Solar activity and the corona, Sol. Phys., 167, 217-265, 1996.

Lundquist, S., Magnetohydrostatic fields, Ark. Fys., 2, 361, 1950.

Martin, S.F., W.H. Marquette, and R. Bilimoria, The solar cycle pattern in the direction of the magnetic field along the long axes of polar filaments, in The Solar Cycle, edited by K.L. Harvey, pp. 53-66, Astron. Soc. of the Pacific, Conf. Ser. 27, San Francisco, CA, 1992. 
Mulligan, T., C.T. Russell, B.J. Anderson, et al., Flux rope modeling of an interplanetary coronal mass ejection observed at WIND and NEAR, in Solar Wind Nine, edited by S.R. Habbal, R. Esser, J.V. Hollweg, and P.A. Isenberg, pp. 689-692, Amer. Inst. Phys. Conf. Proc. 471, Washington, DC, 1999.

Nakagawa, Y., M.A. Raadu, D.E. Billings, et al., On the topology of filaments and chromospheric fibrils near sunspots, Sol. Phys., 19, 72-85, 1971.

Rust, D.M., Magnetic fields in quiescent solar prominences I. Observations, Astrophys. J., 150, 313, 1967.

Rust, D.M., Spawning and shedding helical magnetic fields in the solar atmosphere, Geophys. Res. Lett., 21, 241244, 1994.

Rust, D.M., and A. Kumar, Helicity charging and eruption of magnetic flux from the sun, in Solar Dynamic Phenomena and Solar Wind Consequences, pp. 39-44, ESA, Paris, 1993.

Rust, D.M., and A. Kumar, Helical magnetic fields in filaments, Sol. Phys., 155, 69-97, 1994.

Rust, D.M., and S.F. Martin, A correlation between sunspot whirls and filament type (Abstract), in Solar Active Regions - Comparing Models with Observations, edited by K.S. Balasubramaniam, and G.W. Simon, pp. $337-$ 338, Ast. Soc. Pacific Conf. Ser. 68, San Francisco, CA, 1994.

Seehafer, N., Electric current helicity in the solar atmosphere, Sol. Phys., 125, 219-232, 1990.

Serio, S., G.S. Vaiana, G. Godoli, et al., Configuration and gradual dynamics of prominence-related X-ray coronal cavities, Sol. Phys., 59, 65-86, 1978.

Taylor, J.B., Relaxation of toroidal plasma and generation of reverse magnetic fields, Phys Rev. Lett., 33, 11391141, 1974.

Taylor, J.B., Relaxation and magnetic reconnection in plasmas, Rev. Mod. Phys., 58, 741-763, 1986.

Webb, D.F., R.P. Lepping, L.F. Burlaga, et al., The origin and development of the May 1997 magnetic cloud, $J$. Geophys. Res., 105 (12), 27251-27260, 2000.

E-mail address of D. M. Rust ㅁvid.Rust@jhuapl.edu

Manuscript received 19 October 2002; revised

; accepted 